\title{
INTEGRAL POINTS ON GENERIC FIBERS
}

\author{
ARNAUD BODIN
}

\begin{abstract}
Let $P(x, y)$ be a rational polynomial and $k \in \mathbb{Q}$ be a generic value. If the curve $(P(x, y)=k)$ is irreducible and admits an infinite number of points whose coordinates are integers then there exist algebraic automorphisms that send $P(x, y)$ to the polynomial $x$ or to $x^{2}-d y^{2}, d \in \mathbb{N}$. Moreover for such curves (and others) we give a sharp bound for the number of integral points $(x, y)$ with $x$ and $y$ bounded.
\end{abstract}

\section{INTRODUCTION}

Let $P \in \mathbb{Q}[x, y]$ be a polynomial and $\mathcal{C}=(P(x, y)=0) \subset \mathbb{C}^{2}$ be the corresponding algebraic curve. On old and famous result is the following:

Theorem (Siegel's theorem). Suppose that $\mathcal{C}$ is irreducible. If the number of integral points $\mathcal{C} \cap \mathbb{Z}^{2}$ is infinite then $\mathcal{C}$ is a rational curve.

Our first goal is to prove a stronger version of Siegel's theorem for curve defined by an equation $\mathcal{C}=(P(x, y)=k)$ where $k$ is a generic value. More precisely there exists a finite set $\mathcal{B}$ such that the topology of the complex plane curve $(P(x, y)=k) \subset \mathbb{C}^{2}$ is independent of $k \notin \mathcal{B}$. We say that $k \notin \mathcal{B}$ is a generic value.

Theorem 1. Let $P \in \mathbb{Q}[x, y]$ and let $k \in \mathbb{Q} \backslash \mathcal{B}$ be a generic value. Suppose that the algebraic curve $\mathcal{C}=(P(x, y)=k)$ is irreducible. If $\mathcal{C}$ contains an infinite number of integral points $(m, n) \in \mathbb{Z}^{2}$ then there exists an algebraic automorphism $\Phi \in$ Aut $\mathbb{Q}^{2}$ such

$$
P \circ \Phi(x, y)=x \quad \text { or } \quad P \circ \Phi(x, y)=\alpha\left(x^{2}-d y^{2}\right)+\beta,
$$

where $d \in \mathbb{N}^{*}$ is a non-square and $\alpha \in \mathbb{Q}^{*}, \beta \in \mathbb{Q}$.

In particular the curve $\mathcal{C}=(P(x, y)=k)$ is diffeomorphic to a line $(x=0)$, in which case the set $\mathcal{B}$ is empty or to an hyperbola $x^{2}-d y^{2}=1$ in which case $\mathcal{B}$ is a singleton.

Theorem 1 can be seen as an arithmetic version of the AbhyankarMoh-Suzuki theorem [2] and in fact we use this result. It can also be

Date: November 2, 2018. 
seen as a strong version of a result of Nguyen Van Chau [8] concerning counter-examples to the Jacobian conjecture.

For example, let $P(x, y)=x-y^{d}$. The curve $\mathcal{C}=\left(x-y^{d}=0\right)$ has infinitely many integral points of type $\left(n^{d}, n\right), n \in \mathbb{Z}$. And for the algebraic automorphism $\Phi(x, y)=\left(x+y^{d}, y\right)$ we have $P \circ \Phi(x, y)=x$. In particular the curve $\mathcal{C}$ is send (by $\left.\Phi^{-1}\right)$ to $(x=0)$. As pointing out by Kevin Buzzard the second case corresponds to Pell's equation and for example $\left(x^{2}-2 y^{2}=1\right)$ admits an infinite number of integral points. Of course a kind of reciprocal of Theorem 1 is true. Let $Q_{1}(x, y)=x$, (resp. $\left.Q_{2}(x, y)=x^{2}-d y^{2}\right)$ and $\Phi \in$ Aut $\mathbb{Q}^{2}$ whose inverse $\Phi^{-1}$ as integral coefficients. If we set $P_{1}=Q_{1} \circ \Phi$ (resp. $\left.P_{2}=Q_{2} \circ \Phi\right)$ then the curve $\left(P_{1}=0\right)$ (resp. $\left.\left(P_{2}=1\right)\right)$ has infinitely many integral points.

For non-generic values the result is not true, for example let $P(x, y)=$ $x^{2}-y^{3}$ and $\mathcal{C}=\left(x^{2}-y^{3}=0\right)$. The integral points $\left(n^{3}, n^{2}\right), n \in \mathbb{Z}$ belongs to $\mathcal{C}$, but as $\mathcal{C}$ is singular it cannot be algebraically equivalent to a line.

We will apply Theorem 1 to obtain new bounds for the number of integral points on algebraic curves. Let $\mathcal{C}=(P(x, y)=0)$ be an algebraic curve, and let $d=\operatorname{deg} P$. Let

$$
N(\mathcal{C}, B)=\#\left\{(x, y) \in \mathcal{C} \cap \mathbb{Z}^{2}|| x \mid \leqslant B \text { and }|y| \leqslant B\right\} .
$$

For all irreducible curves Heath-Brown [5] proved that $N(\mathcal{C}, B) \leqslant$ $C_{d} B^{\frac{1}{d}+\varepsilon}$ for some constant $C_{d}$. By making the proof of [5] explicit Walkowiak obtains in [12]:

Theorem (Explicit Heath-Brown's theorem). For all irreducible curve $\mathcal{C}$ of degree $d$ and all $B>0$ :

$$
N(\mathcal{C}, B) \leqslant 2^{48} d^{8} \ln (B)^{5} B^{\frac{1}{d}} .
$$

The term $B^{\frac{1}{d}}$ in Theorem 2 is sharp but the term $2^{48} d^{8} \ln (B)^{5}$ is far from being optimal. For curves $\mathcal{C}$ as in Theorem 1 we will give sharp bounds for $N(\mathcal{C}, B)$. First of all if $\mathcal{C}=(P=k)$ and the polynomial $P$ is algebraically equivalent to $x^{2}-d y^{2}$ it is know $[11$, p. 135] that there exists $C>0$ and $n \geqslant 1$ such that

$$
N(\mathcal{C}, B) \leqslant C \cdot \ln (B)^{n} .
$$

Of course it implies $N(\mathcal{C}, B) \leqslant B^{\frac{1}{d}}$ for sufficiently large $B$ and we shall omit this case.

So if $P$ is not algebraically equivalent to $x^{2}-d y^{2}$ then as a corollary of Theorem 1, such a curve $\mathcal{C}$ admits a parametrisation by polynomials: let $(p(t), q(t))$ be a parametrisation of $\mathcal{C}$ with rational coefficients. 
Moreover $\operatorname{deg} P$ is equal to $\operatorname{deg} p$ or $\operatorname{deg} q$. We suppose $\operatorname{deg} P=\operatorname{deg} p$ and write

$$
p(t)=\frac{1}{b}\left(a_{d} t^{d}+a_{d-1} t^{d-1}+\cdots+a_{0}\right) .
$$

where $a_{0}, \ldots, a_{d}, b \in \mathbb{Z}$ and $\operatorname{gcd}\left(a_{0}, \ldots, a_{d}, b\right)=1$.

Theorem 2. Let $\mathcal{C}$ be as in Theorem 1. Then the number $N(\mathcal{C}, B)$ of integral points on $\mathcal{C}$ bounded by $B$, verifies:

$$
\forall \varepsilon>0 \quad \exists B_{0} \geqslant 0 \quad \forall B \geqslant B_{0} \quad N(\mathcal{C}, B) \leqslant 2 a_{d}^{1-\frac{1}{d}} b^{\frac{1}{d}} B^{\frac{1}{d}}+1+\varepsilon .
$$

In fact by adding a term $\frac{(d-1)(d-2)}{2}$ we prove such a bound for curves $\mathcal{C}$ parametrised by polynomials $(p(t), q(t))$.

For example if $a_{d}=1$ and $b=1$ in Theorem 2 we get for a sufficiently large $B$ :

$$
N(\mathcal{C}, B) \leqslant 2 B^{\frac{1}{d}}+1+\varepsilon .
$$

It implies for $\varepsilon=\frac{1}{2}$ and all sufficiently large $B$ such that $B^{\frac{1}{d}}$ is an integer that we get

$$
N(\mathcal{C}, B) \leqslant 2 B^{\frac{1}{d}}+1
$$

For instance we have a parametrisation of $\mathcal{C}=\left(x-y^{d}=0\right)$ by $\left(t^{d}, t\right)$. If $B^{\frac{1}{d}}$ is an integer we get $N(\mathcal{C}, B)=2 B^{\frac{1}{d}}+1$. Moreover the " $\varepsilon$ " term is necessary as the example $\mathcal{C}=\left(x-y^{d}=1\right)$ proves, see Example 13.

Acknowledgements: This work has been done during a visit at the University of Zaragoza, the author wishes to thank people of the Department of Mathematics and especially Enrique Artal Bartolo for hospitality. I also thank Pierre Dèbes for his support.

\section{PARAmetrisation}

2.1. Topology of polynomials. By a result of Thom for a polynomial $P \in \mathbb{Q}[x, y]$ seen as a map $P: \mathbb{C}^{2} \rightarrow \mathbb{C}$ there exists a finite set $\mathcal{B} \subset \mathbb{C}$ such that

$$
P: P^{-1}(\mathbb{C} \backslash \mathcal{B}) \longrightarrow \mathbb{C} \backslash \mathcal{B}
$$

is a topological locally trivial fibration. A value $k \notin \mathcal{B}$ is a generic value.

For example we have the following characterization of the generic values: the Euler characteristic of the complex plane curve $(P(x, y)=$ $k) \subset \mathbb{C}^{2}$ is independent of $k \notin \mathcal{B}$ and jumps if and only if $k \in \mathcal{B}$.

Of course the image by $P$ of a singular point is not a generic value, but for example $P(x, y)=x(x y-1)$ has no singular points while $\mathcal{B}=$ $\{0\}$. Then if $k \notin \mathcal{B}$ is a generic value the plane algebraic curve $\mathcal{C}=$ 
$(P(x, y)=k) \subset \mathbb{C}^{2}$ does not have singular points. Moreover if $\mathcal{C}$ is connected then $\mathcal{C}$ is irreducible.

The connectedness of a generic fiber $\mathcal{C}$ is equivalent of $P(x, y)$ being non-composite [1]. We recall that $P(x, y)$ is composite if there exist $h \in \mathbb{C}[t], \operatorname{deg} h \geqslant 2$, and $Q \in \mathbb{C}[x, y]$ such that $P(x, y)=h \circ Q(x, y)$. By [3, Theorem 7] we even can choose $h$ and $Q$ with rational coefficients. Consequently it has been noticed by Janusz Gwozdziewicz that the hypothesis " $\mathcal{C}$ is irreducible" in Theorem 1 can be removed. In that case the conclusion becomes $P \circ \Phi(x, y)=h(x)$ or $P \circ \Phi(x, y)=h\left(x^{2}-d y^{2}\right)$, where $h \in \mathbb{Q}[t]$ is a one-variable polynomial of positive degree.

2.2. Algebraic automorphisms. For $K=\mathbb{Q}$ or $K=\mathbb{C}$ an algebraic automorphism $\Phi \in$ Aut $K^{2}$ is a polynomial map $\Phi: K^{2} \longrightarrow K^{2}$, invertible, such that the inverse is also a polynomial map. The polynomials $P, Q \in K[x, y]$ are algebraically equivalent if there exists $\Phi \in$ Aut $K^{2}$ such that $Q=P \circ \Phi$. And in fact such $P$ and $Q$ have the same topological and algebraic properties.

2.3. Siegel's theorem. By Siegel's theorem an irreducible plane algebraic curve $\mathcal{C}$ with an infinite number of integral points $(x, y) \in \mathcal{C} \cap \mathbb{Z}^{2}$ is a rational curve (i.e. the genus is zero).

2.4. Parametrisation. As $\mathcal{C}=(P(x, y)=k)$ is rational it admits a parametrisation by rational fractions. In order to deals with points at infinity and special parameters we compactify the situation.

Let $\bar{P}(x, y, z)$ be the homogenisation of $P(x, y)$ with $d=\operatorname{deg} \bar{P}=$ $\operatorname{deg} P$. Then $\overline{\mathcal{C}}=\left(P(x, y, z)-k z^{d}=0\right) \subset \mathbb{P}^{2}$ is the closure of $\mathcal{C}$.

As $\overline{\mathcal{C}}$ is rational there exists a birational map $\phi: \mathbb{P}^{1} \longrightarrow \overline{\mathcal{C}}$ defined by

$$
\phi(t, s)=(\bar{p}(t, s), \bar{q}(t, s), \bar{r}(t, s))
$$

where $\bar{p}, \bar{q}, \bar{r}$ are homogeneous polynomials of the same degree in $\mathbb{Z}[t, s]$, without common non-constant factor.

We will need some facts about parametrisations (see [9]).

Lemma 3. For any such parametrisation:

(1) $\phi$ is a morphism (it is well-defined everywhere);

(2) $\phi$ is surjective;

(3) $\operatorname{deg} \bar{p}=\operatorname{deg} \bar{q}=\operatorname{deg} \bar{r}=\operatorname{deg} P=d$;

(4) The birational inverse $\psi$ of $\phi$ is well-defined away from the singular points of $\overline{\mathcal{C}}$;

(5) If $(x, y) \in \mathcal{C} \cap \mathbb{Z}^{2}$ is a non-singular point then there exists $(t, s) \in$ $\mathbb{Z}^{2}$ such that $\phi(t, s)=(x: y: 1)$; 
Proof. We just sketch the proofs and we refer to [9, Lemma 2.1] for details and references: the fact that $\bar{p}, \bar{q}, \bar{r}$ have no common factor implies that $\phi$ is well-defined. Then $\phi\left(\mathbb{P}^{1}\right)$ is dense in $\overline{\mathcal{C}}$ and hence is equal to $\overline{\mathcal{C}}$. The birational inverse $\psi$ of $\phi$ is well-defined excepted at the singular points of $\overline{\mathcal{C}}$, see Fulton [4, p.160]. Then a non-singular integral point of $\overline{\mathcal{C}}$ is send by $\psi$ to a point with rational coordinates. We will not need item (3) and we refer to [9].

2.5. Maillet's theorem. An old result of Maillet [6] gives strong restrictions for the parametrisations.

Theorem 4. If $\mathcal{C}=(P(x, y)=k) \subset \mathbb{C}^{2}$ has an infinite number of integral points then there exists a parametrisation $\phi$ of $\overline{\mathcal{C}}$ given by

$$
\phi(t, s)=(\bar{p}(t, s), \bar{q}(t, s), \bar{r}(t, s))
$$

as before with

$$
\bar{r}(t, s)=a t^{d} \quad \text { or } \quad \bar{r}(s, t)=a\left(\alpha t^{2}+\beta t s+\gamma s^{2}\right)^{d / 2}
$$

where $a, \alpha, \beta, \gamma \in \mathbb{Z}$ and $\beta^{2}-4 \alpha \gamma>0$.

\subsection{Topology of $\mathcal{C}$.}

Lemma 5. If $k$ is a generic value and $\mathcal{C}=(P(x, y)=k)$ has an infinite number of integral points then $\mathcal{C}$ is homeomorphic to $\mathbb{C}$ or to $\mathbb{C}^{*}$.

In fact the homeomorphisms are diffeomorphisms and we only need $k$ to be a non-singular value of $P(x, y)$. In Lemma 9 we will exclude the case $\mathbb{C}^{*}$.

Proof. Let $L_{\infty}=(z=0)$ of $\mathbb{P}^{2}$ be the line at infinity. What are the parameters $(t: s)$ that give the points at infinity $\overline{\mathcal{C}} \cap L_{\infty}$ ? The points at infinity correspond to the parameters $(t: s)$ such that $\bar{r}(s, t)=0$.

So if $\phi$ is the parametrisation given by Maillet's theorem then for $\bar{r}(t, s)=a t^{d}$ then $(t: s)=(0: 1)$ and there is one point $P \in \overline{\mathcal{C}} \cap L_{\infty}$. For $\bar{r}(s, t)=a\left(\alpha t^{2}+\beta t s+\gamma s^{2}\right)^{d / 2}$ then $(t: s)=\left(\tau_{1}: 1\right)$ or $(t, s)=\left(\tau_{2}: 1\right)$ where $\tau_{1}, \tau_{2}$ are the roots of $\alpha t^{2}+\beta t+\gamma($ say $\alpha \neq 0)$; there are two points $P_{1}, P_{2} \in \overline{\mathcal{C}} \cap L_{\infty}$.

From Lemma 3 we know that $\phi$ is morphism that induces a bijective map onto $\overline{\mathcal{C}} \backslash \operatorname{Sing} \overline{\mathcal{C}}$. The only singular points of $\overline{\mathcal{C}}$ are on the line at infinity $L_{\infty}$ because $\mathcal{C}$ is a non-singular affine curve. Then in the case $\bar{r}(s, t)=a t^{d}$, the restriction $\phi_{\mid}: \mathbb{P}^{1} \backslash\{(0: 1)\} \longrightarrow \overline{\mathcal{C}} \backslash\{P\}$ is a bijective map. Moreover it is an homeomorphism. But $\mathbb{P}^{1} \backslash\{(0: 1)\}=\mathbb{C}$ and $\overline{\mathcal{C}} \backslash\{P\}=\mathcal{C}$. Then $\mathcal{C}$ is homeomorphic to $\mathbb{C}$.

In the case $\bar{r}(s, t)=a\left(\alpha t^{2}+\beta t s+\gamma s^{2}\right)^{d / 2}$, the restriction $\phi_{1}: \mathbb{P}^{1} \backslash\left\{\left(\tau_{1}:\right.\right.$ $\left.1),\left(\tau_{2}: 1\right)\right\} \longrightarrow \overline{\mathcal{C}} \backslash\left\{P_{1}, P_{2}\right\}$ is an homeomorphism from $\mathbb{P}^{1} \backslash\left\{\left(\tau_{1}:\right.\right.$ 1), $\left.\left(\tau_{2}: 1\right)\right\}$ to $\mathcal{C}=\overline{\mathcal{C}} \backslash\left\{P_{1}, P_{2}\right\}$. Then $\mathcal{C}$ is homeomorphic to $\mathbb{C}^{*}$. 
2.7. Case of $\mathcal{C}$ being homeomorphic to $\mathbb{C}$. We recall the AbhyankarMoh-Suzuki [1, 2, 10] theorem:

\section{Theorem 6.}

(1) Let $t \mapsto(p(t), q(t))$ be an injective polynomial map over $\mathbb{C}$ such that the tangent vector $\left(p^{\prime}(t), q^{\prime}(t)\right)$ is never $(0,0)$ then $\operatorname{deg} p$ divides $\operatorname{deg} q$, or $\operatorname{deg} q$ divides $\operatorname{deg} p$.

(2) Let $\mathcal{C}=(P(x, y)=0) \subset \mathbb{C}^{2}$ be an algebraic plane curve nonsingular and homeomorphic to $\mathbb{C}$ then there exists an algebraic automorphism $\Phi \in$ Aut $\mathbb{C}^{2}$ such that

$$
P \circ \Phi(x, y)=x \text {. }
$$

The second statement is the usual form of the Abhyankar-MohSuzuki theorem, it is in fact a consequence of the first (see the proof below), for which a more general statement exists [2].

Lemma 7. If $\mathcal{C}=(P(x, y)=k), k$ a generic value, is homeomorphic to $\mathbb{C}$ then there exists $\Phi \in$ Aut $\mathbb{Q}^{2}$ whose inverse has integral coefficients such that $P \circ \Phi(x, y)=a x+b, a, b \in \mathbb{Q}$.

Proof. The existence of $\Phi \in$ Aut $\mathbb{C}^{2}$ is Theorem 6-(2). But here we ask the coefficients of $\Phi$ to be rationals and those of $\Phi^{-1}$ to be integers: we will apply Theorem 6 -(1). As $\mathcal{C}$ is homeomorphic to $\mathbb{C}$ let $(p(t), q(t))$ be a parametrisation of $\mathcal{C}$ with $p(t), q(t) \in \mathbb{Q}[t]$ (it comes from setting $s=1$ in a parametrisation $\phi=\left(\bar{p}(t, s), \bar{q}(t, s), a s^{d}\right)$ of $\left.\overline{\mathcal{C}}\right)$. Then $\operatorname{deg} p$ divides $\operatorname{deg} q$ or $\operatorname{deg} q$ divides $\operatorname{deg} p$. Suppose that $\delta=\operatorname{deg} p>0$ divides $\operatorname{deg} q$ and write $p(t)=a_{\delta} t^{\delta}+a_{\delta-1} t^{\delta-1}+\cdots$ and $q(t)=b_{\ell \delta} t^{\ell \delta}+b_{\ell \delta-1} t^{\ell \delta-1}+\cdots$ with $a_{i}, b_{i} \in \mathbb{Q}$ and $\ell \geqslant 1$. Write $a_{\delta}=\frac{\alpha}{\beta}$ and $b_{\ell \delta}=\frac{\alpha^{\prime}}{\beta^{\prime}}$. Set the algebraic automorphism of Aut $\mathbb{Q}^{2}$,

$$
\Phi_{1}(x, y)=\left(\frac{1}{\alpha \beta} x, \frac{1}{\alpha} \ell y-\frac{\beta^{\ell}}{\alpha^{\ell} \beta^{\prime}} x^{\ell}\right),
$$

whose inverse is

$$
\Phi_{1}^{-1}(x, y)=\left(\alpha \beta^{\prime} x, \alpha^{\prime} y+\alpha^{\prime} \beta^{\prime \ell-1} \beta^{\ell} x^{\ell}\right),
$$

whose coefficients are integers.

The composition with $\Phi_{1}$ yields a parametrisation of $(P-k) \circ \Phi_{1}(x, y)$ given by $p(t), q^{\prime}(t)$ with $q^{\prime}(t) \in \mathbb{Q}[t]$ and $\operatorname{deg} q^{\prime}<\operatorname{deg} q$. We repeat this process until one of $p(t)$ or $q(t)$ is a constant the other being of degree 1 (because $\mathcal{C}$ does not have singular points). Then by the algebraic automorphism $\Phi=\Phi_{1} \circ \Phi_{2} \circ \cdots$, whose inverse has integral coefficients, we get $(P-k) \circ \Phi(x, y)=a x+b, a, b \in \mathbb{Q}$. 
2.8. Case of $\mathcal{C}$ being homeomorphic to $\mathbb{C}^{*}$. We will need the classification over $\mathbb{C}$ of polynomials with a generic fiber homeomorphic to $\mathbb{C}^{*}$, due to $W$. Neumann $[7, \S 8]$.

Theorem 8. If $\mathcal{C}=(P(x, y)=k), k \neq 0$ a generic value, is homeomorphic to $\mathbb{C}^{*}$ then $P$ there exists an algebraic automorphism $\Phi \in$ Aut $\mathbb{C}^{2}$ such that

$$
\begin{gathered}
P \circ \Phi(x, y)=x^{p} y^{q}+\beta \\
\text { or } P \circ \Phi(x, y)=x^{p}\left(y x^{r}+a_{r-1} x^{r-1}+\cdots+a_{0}\right)^{q}+\beta,
\end{gathered}
$$

with $\beta \in \mathbb{C}, p>0, q>0, \operatorname{gcd}(p, q)=1, r>0, a_{0}, \ldots, a_{r-1} \in \mathbb{C}$ and $a_{0} \neq 0$.

We will prove that only some special polynomials of the first type can have an infinite number of integral points.

The main result of this part is the following lemma.

Lemma 9. If $\mathcal{C}=(P(x, y)=k), k$ a generic value, is homeomorphic to $\mathbb{C}^{*}$ with an infinite number of integral points then there exists $\Phi \in$ Aut $\mathbb{Q}^{2}$ such that $P \circ \Phi(x, y)=\alpha\left(x^{2}-d y^{2}\right)+\beta, \alpha \in \mathbb{Q}^{*}, \beta \in \mathbb{Q}$.

Proof. By Theorem 8 we know that the polynomial $P$ has exactly two absolute irreducible factors. For simplicity of the redaction we suppose in the following that $\beta=0$.

First case : $P$ is reducible in $\mathbb{Q}[x, y]$.

Once again we will prove that $\Phi$ of Theorem 8 can be chosen with rational coefficients and its inverse with integral coefficients. We write $P=\alpha A^{p} B^{q}$ the decomposition into irreducible factors with $A, B \in$ $\mathbb{Q}[x, y]$. Again for simplicity we suppose $\alpha=1$. We will decompose the proof according to the cases of Theorem 8 . In both cases we see that the curve $(P=0)$ has a non-singular irreducible component homeomorphic to $\mathbb{C}$ (the one send by $\Phi^{-1}$ to $\left.(x=0)\right)$. Notice that this curve $(P=0)$ is not the curve $\mathcal{C}$. This component homeomorphic to $\mathbb{C}$ is either $(A=0)$ or $(B=0)$, say it is $(A=0)$. Then, as $A \in \mathbb{Q}[x, y]$, by the version of Abhyankar-Moh-Suzuki theorem used as in Lemma 7 above, there exists $\Psi \in$ Aut $\mathbb{Q}^{2}$, whose inverse has integral coefficients, such that: $A \circ \Psi(x, y)=a x+b$, this implies :

$$
P \circ \Psi(x, y)=(a x+b)^{p} Q(x, y)^{q} .
$$

Sub-case $P \circ \Phi(x, y)=x^{p} y^{q}$.

Then $(Q(x, y)=0)$ is non-singular, homeomorphic to $\mathbb{C}$ and the intersection multiplicity with $a x+b$ is 1 . Then if $(p(t), q(t))$ is a polynomial parametrisation of $(Q(x, y)=0)$ we have $\operatorname{deg} p=1$. Then as in the proof of Lemma 7 by algebraic automorphisms of type $(x, y) \mapsto$ 
$\left(\alpha x, \beta y-\gamma x^{\ell}\right)$ whose inverse have integral coefficients, we can suppose that $q(t)$ is a constant. Notice that such automorphisms preserve vertical lines.

Then $Q(x, y)$ becomes $c y+d, c, d \in \mathbb{Q}$, while $a x+b$ remains unchanged. Then we have find $\Psi^{\prime} \in$ Aut $\mathbb{Q}^{2}$ whose inverse has integral coefficients such that:

$$
P \circ \Psi^{\prime}(x, y)=(a x+b)^{p}(c y+d)^{q} .
$$

Sub-case $P \circ \Phi(x, y)=x^{p}\left(y x^{r}+a_{r-1} x^{r-1}+\cdots+a_{0}\right)^{q}$.

$P \circ \Phi(x, y)=x^{p}\left(y x^{r}+a_{r-1} x^{r-1}+\cdots+a_{0}\right)^{q}$ is algebraically equivalent to $P \circ \Psi(x, y)=(a x+b)^{p} Q(x, y)^{q}$ by the algebraic automorphism $\Phi \circ \Psi^{-1}$. Moreover $\Phi \circ \Psi^{-1}$ should send $x$ to $a x+b$. Then $\Phi \circ \Psi^{-1}$ is the composition of algebraic automorphisms of type $(x, y) \mapsto(a x+b, y)$ and $(x, y) \mapsto\left(\alpha x, \beta y-\gamma x^{\ell}\right)$. This implies that the degree in the variable $y$ remains unchanged. Then $\operatorname{deg}_{y} Q(x, y)=\operatorname{deg}_{y}\left(y x^{r}+a_{r-1} x^{r-1}+\right.$ $\left.\cdots+a_{0}\right)=1$. Then $Q(x, y)=q_{1}(x) y+q_{2}(x)$. Due to the asymptotic branches we have $q_{1}(x, y)=(a x+b)^{r}$. And by algebraic automorphisms whose inverse have integral coefficients of type $(x, y) \mapsto\left(\alpha x, \beta y-\gamma x^{\ell}\right)$ we can suppose $\operatorname{deg} q_{2}<r$. Then we have found $\Psi^{\prime} \in$ Aut $\mathbb{Q}^{2}$ with an inverse having integral coefficients such that:

$$
P \circ \Psi^{\prime}(x, y)=(a x+b)^{p}\left(y(a x+b)^{r}+b_{r-1} x^{r-1}+\cdots+b_{0}\right)^{q},
$$

$b_{0}, \ldots, b_{r-1} \in \mathbb{Q}, b_{0} \neq 0$.

\section{Conclusion for both sub-cases.}

Now the curve $\left(P \circ \Psi^{\prime}(x, y)=k\right)$ has a finite number of integral points since the branches at infinity are asymptotic to horizontal or vertical lines (with equation $(a x+b=0),(c y+d=0)$ in the first case and $(a x+b=0),(y=0)$ in the second case). Now as $\Psi^{\prime-1}$ has integral coefficients, an integral point $(m, n) \in(P(x, y)=k) \cap \mathbb{Z}^{2}$ is sent to an integral point $\Psi^{\prime-1}(m, n) \in\left(P \circ \Psi^{\prime}(x, y)=k\right) \cap \mathbb{Z}^{2}$ it implies that $\mathcal{C}=(P(x, y)=k)$ also have a finite number of integral points.

Second case : $P$ is irreducible in $\mathbb{Q}[x, y]$.

Then by Lemma 10 below it implies that there exist $D, E \in \mathbb{Q}[x, y]$, $d \in \mathbb{Z}$ such that $P=C^{2}-d D^{2}$. Then $P=(C-\sqrt{d} D)(C+\sqrt{d} D)$ is the decomposition into irreducible factors.

Sub-case $P \circ \Phi(x, y)=x^{p} y^{q}$.

The by Lemma 10 we know that $p=1, q=1$. And equivalently there exists $\Phi^{\prime} \in$ Aut $\mathbb{C}^{2}$ such that $P \circ \Phi^{\prime}(x, y)=(x-\sqrt{d} y)(x+\sqrt{d} y)$. Then $P \circ \Phi^{\prime}(x, y)=\left(C^{2}-d D^{2}\right) \circ \Phi^{\prime}(x, y)=(x-\sqrt{d} y)(x+\sqrt{d} y)$. We may suppose that $(C-\sqrt{d} D) \circ \Phi^{\prime}(x, y)=(x-\sqrt{d} y)$ and $(C+$ $\sqrt{d} D) \circ \Phi^{\prime}(x, y)=(x+\sqrt{d} y)$, by addition and subtraction we get 
$C \circ \Phi^{\prime}(x, y)=x$ and $D \circ \Phi^{\prime}(x, y)=y$. Then $(C D) \circ \Phi^{\prime}(x, y)=x y$, with $C, D \in \mathbb{Q}[x, y]$. As in the first case above we are now enable to find $\Psi \in$ Aut $\mathbb{Q}^{2}$ (whose inverse as integral coefficients) such that $C \circ \Psi(x, y)=x, D \circ \Psi(x, y)=y$ and $C D \circ \Psi(x, y)=x y$. Now $P \circ \Psi(x, y)=\left(C^{2}-d D^{2}\right) \circ \Psi(x, y)=x^{2}-d y^{2}$.

Sub-case $P \circ \Phi(x, y)=x^{p}\left(y x^{r}+a_{r-1} x^{r-1}+\cdots+a_{0}\right)^{q}$.

Again $p=1, q=1$, and we may suppose that $(C-\sqrt{d} D) \circ \Phi(x, y)=$ $x$. We denote $Q=y \circ \Phi^{-1}(x, y)$ i.e. $Q \circ \Phi(x, y)=y$. Then

$$
\begin{aligned}
(C-\sqrt{d} D)(C+\sqrt{d} D) & =P \\
& =x\left(y x^{r}+a_{r-1} x^{r-1}+\cdots+a_{0}\right) \circ \Phi^{-1}(x, y) \\
& =(C-\sqrt{d} D)\left(Q(C-\sqrt{d} D)^{r}+\cdots\right)
\end{aligned}
$$

Then

$$
(C+\sqrt{d} D)=\left(Q(C-\sqrt{d} D)^{r}+\cdots\right) .
$$

But as $C, D \in \mathbb{Q}[x, y]$ we have $d=\operatorname{deg}(C+\sqrt{d} D)=\operatorname{deg}(C-\sqrt{d} D)$ and we get $d=\operatorname{deg} Q+r d$. As $r \geqslant 1$ we get $\operatorname{deg} Q=0$ which is in contradiction with the definition of $Q$. Then this sub-case does not occur.

Lemma 10. Let $P \in \mathbb{Q}[x, y]$ such that $P=\alpha A^{p} B^{q}$, with $\operatorname{gcd}(p, q)=1$ and with $\alpha \in \mathbb{Q}^{*}, A, B \in \overline{\mathbb{Q}}[x, y]$ normalized and irreducible (that is to say $P$ admits exactly two absolute irreducible factors). Then either $A, B \in \mathbb{Q}[x, y]$ or $p=1, q=1$ and there exist $C, D \in \mathbb{Q}[x, y], d \in \mathbb{Z}$ non-square such that $P=\alpha\left(C^{2}-d D^{2}\right)$.

The following proof is due to Pierre Dèbes.

Proof. Let $a_{i, j} \in \overline{\mathbb{Q}}$ be the coefficients of $A$. Let $n$ be the degree of the finite extension $\mathbb{Q}\left(\left(a_{i, j}\right)\right) / \mathbb{Q}$. Then there exist exactly $n$ distinct conjugates of $A$. But for all $\sigma \in \operatorname{Gal}(\overline{\mathbb{Q}} / \mathbb{Q}), \sigma(A) \in\{A, B\}$. Then $A$ has at most two distinct algebraic conjugates. Thus $n=1$ or $n=2$. If $A \notin \mathbb{Q}[x, y]$ then there exists $a_{i_{0}, j_{0}} \notin \mathbb{Q}$ and a $\sigma_{0} \in \operatorname{Gal}(\overline{\mathbb{Q}} / \mathbb{Q})$ such that $\sigma_{0}(A)=B$. Then $n=2$ so that the extension $\mathbb{Q}\left(\left(a_{i, j}\right)\right) / \mathbb{Q}$ is quadratic. Moreover $p=q=1$. This implies the existence of a non-square integer $d$ such that $A, B \in \mathbb{Q}(\sqrt{d})[x, y]$. Now if we write $A=C+\sqrt{d} D$, $C, D \in \mathbb{Q}[x, y]$ then its algebraic conjugate is $B=C-\sqrt{d} D$.

Lemma 7 and Lemma 9 imply Theorem 1 of the introduction. 


\section{Number of INTEGRAL POINTS ON POLYNOMials CURVES}

Lemma 11. Let $p(t)=a_{d} t^{d}+a_{d-1} t^{d-1}+\cdots+a_{0} \in \mathbb{Q}[t], a_{d}>0$. Let $\sigma=-\frac{a_{d-1}}{d a_{d}}$. For all $\varepsilon>0$ there exists $B_{0}>0$ such that for all $B \geqslant B_{0}$ and

$$
t_{+}=\left(\frac{B}{a_{d}}\right)^{\frac{1}{d}}+\sigma+\varepsilon, \quad t_{-}=-\left(\frac{B}{a_{d}}\right)^{\frac{1}{d}}+\sigma-\varepsilon
$$

then

$$
\forall t \geqslant t_{+} \quad|p(t)|>B \quad \text { and } \quad \forall t \leqslant t_{-} \quad|p(t)|>B .
$$

A similar result holds if $a_{d}<0$.

Proof. We write $t=s+\sigma+\varepsilon$ and we look at the asymptotic behaviour for $p(t)$ when $t$ (and $s$ ) is large.

$$
\begin{aligned}
p(t) & =p(s+\sigma+\varepsilon) \\
& =a_{d}(s+\sigma+\varepsilon)^{d}+a_{d-1}(s+\sigma+\varepsilon)^{d-1}+\cdots \\
& =a_{d} s^{d}+\left(d a_{d}(\sigma+\varepsilon)+a_{d-1}\right) s^{d-1}+o\left(s^{d-1}\right) \\
& =a_{d} s^{d}+d a_{d} \varepsilon s^{d-1}+o\left(s^{d-1}\right) .
\end{aligned}
$$

For $s=\left(\frac{B}{a_{d}}\right)^{\frac{1}{d}}$ then $a_{d} s^{d}=B$ we have

$$
\begin{aligned}
p\left(t_{+}\right) & =p(s+\sigma+\varepsilon) \\
& =B \cdot\left(1+\varepsilon d \frac{1}{s}+o\left(\frac{1}{s}\right)\right) .
\end{aligned}
$$

Then for all sufficiently large $B$ (such that $s>0$ is large enough) we have $p\left(t_{+}\right) \geqslant B\left(1+\frac{\varepsilon d}{2} \frac{1}{s}\right)$ then $p\left(t_{+}\right)>B$. Now the function $t \mapsto p(t)$ is an increasing function for sufficiently large $t$. Then for all sufficiently large $B$ : if $t \geqslant t_{+}$then $p(t) \geqslant p\left(t_{+}\right)>B$.

Now

$$
\begin{aligned}
p\left(t_{-}\right) & =p(-s+\sigma-\varepsilon) \\
& =(-1)^{d} B \cdot\left(1+\varepsilon d \frac{1}{s}+o\left(\frac{1}{s}\right)\right) .
\end{aligned}
$$

Then for all sufficiently large $B,\left|p\left(t_{-}\right)\right|>B$. And again if $t<t_{-}$then $|p(t)| \geqslant\left|p\left(t_{-}\right)\right|>B$.

For a polynomial $p(t) \in \mathbb{Q}[t]$ in one variable we defined:

$$
M(p, B)=\{t \in \mathbb{Q} \mid p(t) \in \mathbb{Z} \text { and }|p(t)| \leqslant B\} .
$$


Lemma 12. Let $p(t)=\frac{1}{b}\left(a_{d} t^{d}+\cdots+a_{0}\right) \in \mathbb{Q}[t], a_{0}, \ldots, a_{d}, b \in \mathbb{Z}$, $\operatorname{gcd}\left(a_{0}, \ldots, a_{d}, b\right)=1$ and $a_{d}>0$. Then for all $\varepsilon>0$ there exists $B_{0}>0$ such that for all $B \geqslant B_{0}$ we have

$$
M(p, B) \leqslant 2 a_{d}^{1-\frac{1}{d}} b^{\frac{1}{d}} B^{\frac{1}{d}}+1+\varepsilon .
$$

Proof. If $t=\frac{\alpha}{\beta} \in \mathbb{Q}$ and $p\left(\frac{\alpha}{\beta}\right)=k \in \mathbb{Z}$ then it is well-known that $\beta$ divides $a_{d}$. Then such $t$ belongs to $\frac{1}{a_{d}} \mathbb{Z}$. Let $\varepsilon>0$ and let $B_{0}$ be as in Lemma 11. Again by Lemma 11 if $t>0$ and $|p(t)| \leqslant B$ then $t<t_{+}=\left(\frac{B}{a_{d} / b}\right)^{\frac{1}{d}}+\sigma+\varepsilon$. If $t<0$ and $|p(t)| \leqslant B$ then $|t|<\left|t_{-}\right|=$ $-t_{-}=\left(\frac{B}{a_{d} / b}\right)^{\frac{1}{d}}-\sigma+\varepsilon$. Now the cardinal of $\frac{1}{a_{d}} \mathbb{Z} \cap\left[t_{-}, t_{+}\right]$is less than $a_{d}\left|t_{+}\right|+a_{d}\left|t_{-}\right|+1=2 a_{d}\left(\frac{B}{a_{d} / b}\right)^{\frac{1}{d}}+\sigma-\sigma+2 \varepsilon+1=2 a_{d}\left(\frac{b B}{a_{d}}\right)^{\frac{1}{d}}+1+2 \varepsilon$.

Of course if $p(t)$ is a monic polynomial with integral coefficients, i.e. $b=1, a_{d}=1$, then $M(p, B) \leqslant 2 B^{\frac{1}{d}}+1+\varepsilon$. For example if $p(t)=t^{d}$ then $M(p, B)=2 B^{\frac{1}{d}}+1$. The following examples show that the " $\varepsilon$ " is necessary and that the bound of Lemma 12 is the best one (at least for $a_{d}=1$ ).

Example 13. Let $p(t)=t^{d}-1$ where $d$ is an even number. Then the following assertion:

$$
\exists B_{0}>0 \quad \forall B \geqslant B_{0} \quad M(p, B) \leqslant 2 B^{\frac{1}{d}}+1 \quad \text { is false. }
$$

In fact for $k$ any positive integer, set $B_{k}=p(k)=k^{d}-1$. Then as $d$ is even for all $t \in[-k, k]$ we have $t^{d}-1 \leqslant k^{d}-1=B_{k}$ then $M\left(p, B_{k}\right)=2 k+1$. Now if the assertion were true we would have $M\left(p, B_{k}\right) \leqslant 2 B_{k}^{\frac{1}{d}}+1$ that it to say $2 k+1 \leqslant 2 p(k)^{\frac{1}{d}}+1$, then $k^{d} \leqslant$ $p(k)=k^{d}-1$ which gives the contradiction.

Here is another example.

Example 14. Let $p(t)=\frac{1}{d !}(t-1)(t-2) \cdots(t-d)$ then for all $t \in$ $\mathbb{Z}$ we have $p(t) \in \mathbb{Z}$. Conversely if $p\left(\frac{\alpha}{\beta}\right) \in \mathbb{Z}$ then $\beta=1$. Then for a positive integer $k$ and $B_{k}=p(k),|p(-k+d)| \leqslant B_{k}$. we have $M\left(p, B_{k}\right) \geqslant 2 k+1-d$. Lemma 12 applied with $a_{d}=1$ and $b=d$ ! gives $M(p, B) \leqslant 2(d !)^{\frac{1}{d}} B_{k}^{\frac{1}{d}}+1+\varepsilon$. Now $B_{k}=p(k) \leqslant \frac{k^{d}}{d !}$ and we get

$$
2 k+1-d \leqslant M\left(p, B_{k}\right) \leqslant 2 k+1+\varepsilon .
$$

We apply these computations to the situation of our curves.

Let $P(x, y) \in \mathbb{Q}[x, y]$ be irreducible, let $\mathcal{C}=(P(x, y)=0)$. Then $\mathcal{C}$ is a polynomial curve if it admits a polynomial parametrisation 
$(p(t), q(t)), p(t), q(t) \in \mathbb{Q}[t]$. Equivalently $\mathcal{C}$ is a rational curve with one place at infinity. Moreover $\operatorname{deg} P=\max (\operatorname{deg} p, \operatorname{deg} q)$. We will suppose $\operatorname{deg} P=\operatorname{deg} p$ and we write $p(t)=\frac{1}{b}\left(a_{d} t^{d}+\cdots+a_{0}\right)$ as before.

Lemma 15. Let $\mathcal{C}$ be a polynomial curve. Suppose $\operatorname{deg} P=d=\operatorname{deg} p$, $p(t)=\frac{1}{b}\left(a_{d} t^{d}+\cdots+a_{0}\right)$. Then for all $\varepsilon>0$ there exists $B_{0}>0$ such that for all $B \geqslant B_{0}$ :

$$
N(\mathcal{C}, B) \leqslant 2 a_{d}^{1-\frac{1}{d}} b^{\frac{1}{d}} B^{\frac{1}{d}}+\frac{(d-1)(d-2)}{2}+1+\varepsilon .
$$

The term $\frac{(d-1)(d-2)}{2}$ comes from the number of singular points; for non-singular curves we get the bound of Theorem 2 .

Proof. An algebraic curve of degree $d$ must have less than $\frac{(d-1)(d-2)}{2}$ singular points, see [4, p.117]. The other integral points $(p(t), q(t))$ of $\mathcal{C}$ correspond to rational parameters $t$, see Lemma 3. Now we apply Lemma 12.

Examples 13 and 14 give polynomials parametrised by $(p(t), t)$ that proves that the bound of Lemma 15 is asymptotically sharp.

\section{REFERENCES}

[1] E. Artal-Bartolo, Une démonstration géométrique du théorème d'AbhyankarMoh. J. Reine Angew. Math. 464 (1995), 97-108.

[2] S.S. Abhyankar, T.T. Moh, Embeddings of the line in the plane. J. Reine Angew. Math. 276 (1975), 148-166.

[3] M. Ayad, Sur les polynômes $f(X, Y)$ tels que $K[f]$ est intégralement fermé dans $K[X, Y]$. Acta Arith. 105 (2002), 9-28.

[4] W. Fulton, Algebraic curves. Addison-Wesley, reprint of 1969.

[5] D.R. Heath-Brown, The density of rational points on curves and surfaces. Ann. of Math. 155 (2002), 553-595.

[6] E. Maillet, Détermination des points entiers des courbes algébriques unicursales à coefficients entiers. J. Ecole Polytech. 2, 20 (1919), 115-156.

[7] W.D. Neumann, Complex algebraic plane curves via their links at infinity. Invent. Math. 98 (1989), 445-489.

[8] Nguyen Van Chau, Integer points on a curve and the plane Jacobian problem. Ann. Polon. Math. 88 (2006), 53-58.

[9] D. Poulakis, E. Voskos, On the practical solution of genus zero Diophantine equations. J. Symbolic Comput. 30 (2000), 573-582.

[10] L. Rudolph, Embeddings of the line in the plane. J. Reine Angew. Math. 337 (1982), 113-118.

[11] J.-P. Serre, Lectures on the Mordell-Weil theorem. Aspects of Mathematics, Vieweg 1997.

[12] Y. Walkowiak, Théorème d'irréductibilité de Hilbert effectif. Acta Arith. 116 (2005), 343-362.

Laboratoire Paul Painlevé, Mathématiques, Université de Lille 1, 59655 Villeneuve D'AscQ, France.

E-mail address: Arnaud.Bodin@math.univ-lille1.fr 